\title{
FrustratometeR: an R-package to compute local frustration in protein structures, point mutants and MD simulations
}

\author{
Atilio O. Rausch ${ }^{1 *}$, Maria I. Freiberger ${ }^{2 *}$, Cesar O. Leonetti ${ }^{2}$, Diego M. Luna ${ }^{1}$, Leandro G. Radusky ${ }^{3}$, Peter G. Wolynes ${ }^{4}$, Diego \\ U. Ferreiro ${ }^{2}$, and R. Gonzalo Parra ${ }^{5 凶}$ \\ ${ }^{1}$ Facultad de Ingeniería, Universidad Nacional de Entre Ríos, Argentina., ${ }^{2}$ Laboratorio de Fisiología de Proteínas, Departamento de Química Biológica - \\ IQUIBICEN/CONICET, Facultad de Ciencias Exactas y Naturales, Universidad de Buenos Aires, Argentina., ${ }^{3}$ Center for Genomic Regulation, Barcelona Institute for \\ Science and Technology, Barcelona, Spain., ${ }^{4}$ Center for Theoretical Biological Physics and Department of Chemistry, Rice University, Houston, TX, 77005, USA., ${ }^{5}$ \\ European Molecular Biology, Laboratory, Heidelberg, Germany., " Jointly 1st authors
}

\begin{abstract}
Once folded, natural protein molecules have few energetic conflicts within their polypeptide chains. Many protein structures do however contain regions where energetic conflicts remain after folding, i. e. they have highly frustrated regions. These regions, kept in place over evolutionary and physiological timescales, are related to several functional aspects of natural proteins such as protein-protein interactions, small ligand recognition, catalytic sites and allostery. Here we present Frustratomete $R$, an $R$ package that easily computes local energetic frustration on a personal computer or a cluster. This package facilitates large scale analysis of local frustration, point mutants and molecular dynamics (MD) trajectories, allowing straightforward integration of local frustration analysis into pipelines for protein structural analysis.
\end{abstract}

Contact: gonzalo.parra@embl.de

Availability and implementation:

https://github.com/proteinphysiologylab/frustratometeR

R package | protein structures | local frustration | molecular dynamics Correspondence: gonzalo.parra@embl.de

\section{Introduction}

Proteins are evolved biological molecules that adopt a defined set of structures constituting their 'native' state. Built as linear polymers, proteins find their native state easily since evolution has minimized the internal energetic conflicts within their polypeptide chain, following the "principle of minimal frustration" (1). Proteins are not only biologically optimized to fold or to be stable but also to 'function' $(2,3)$ and therefore it's not surprising to find that about $10-15 \%$ of the internal interactions in proteins are in energetic conflict within their local structure (4). These conflicts, kept in place over evolutionary and physiological time scales, allow proteins to explore different conformations within their native ensemble and thus enable the emergence of 'function'. Over the last years, the concept of local frustration has given insights into a diverse set of functional phenomena: protein-protein interactions, ligand recognition, allosteric sites (5), enzymatic active sites and co-factors binding (6), evolutionary patterns in protein families (7), polymorphisms in the Class I Major Histocompatibility Complex (MHC) (8), disease associated mutations (9), protein dynam- ics (10), disorder-to-order transitions in protein complexes $(11,12)$ as well as fuzzy interactions (13) and chaperonesclients recognition (14). Frustration and its role in functional dynamics has been recently reviewed (15). Up to now, facile location and quantification of energetic frustration has been made possible via the Frustratometer web server $(16,17)$. Unfortunately high-throughput analysis using the server is not feasible as the flexibility of the algorithm was reduced in order to maximise usability by non computational scientists. Here we present FrustratometeR, an R package that retains all the capabilities present in the web server but that also includes brand new modules to evaluate how frustration varies upon point mutations as well as to analyse how frustration varies as a function of time during molecular dynamics simulations. FrustratometeR facilitates the analysis both at small and high-throughput scales and thus allows one to integrate local frustration analysis into other protein structural bioinformatics pipelines.

\section{Methods}

Full methods descriptions are available in the supplementary material. Fig1 summarises FrustratometeR functionalities and minimum code to generate plots there in (Fig1E). Here we describe the main functions in the package. The three functions can be used in combination with any of the frustration indexes (i.e. configurational, mutational or singleresidue). If not specified the configurational mode is used as default.

Calculate local frustration: A single function, calculate frustration(), the PDB structure or PDBID and the desired frustration index specification are enough to calculate the local frustration either for single (Fig1A) or multiple chain structures (Fig1B). Optionally an electrostatic term can be activated in the energy function by giving a value to the Electrostatics_K parameter that is set by default to NULL (see supplementary material or (17) for details).

Frustration upon mutations: The mutate_res() function allows users to analyse how point mutations affect local frustration (Fig1C). For every possible alternative amino acid, this function generates a structural variant. Two modes are implemented to generate the mutants: threading (it does not modify the backbone coordinates) and modeller (performs some 

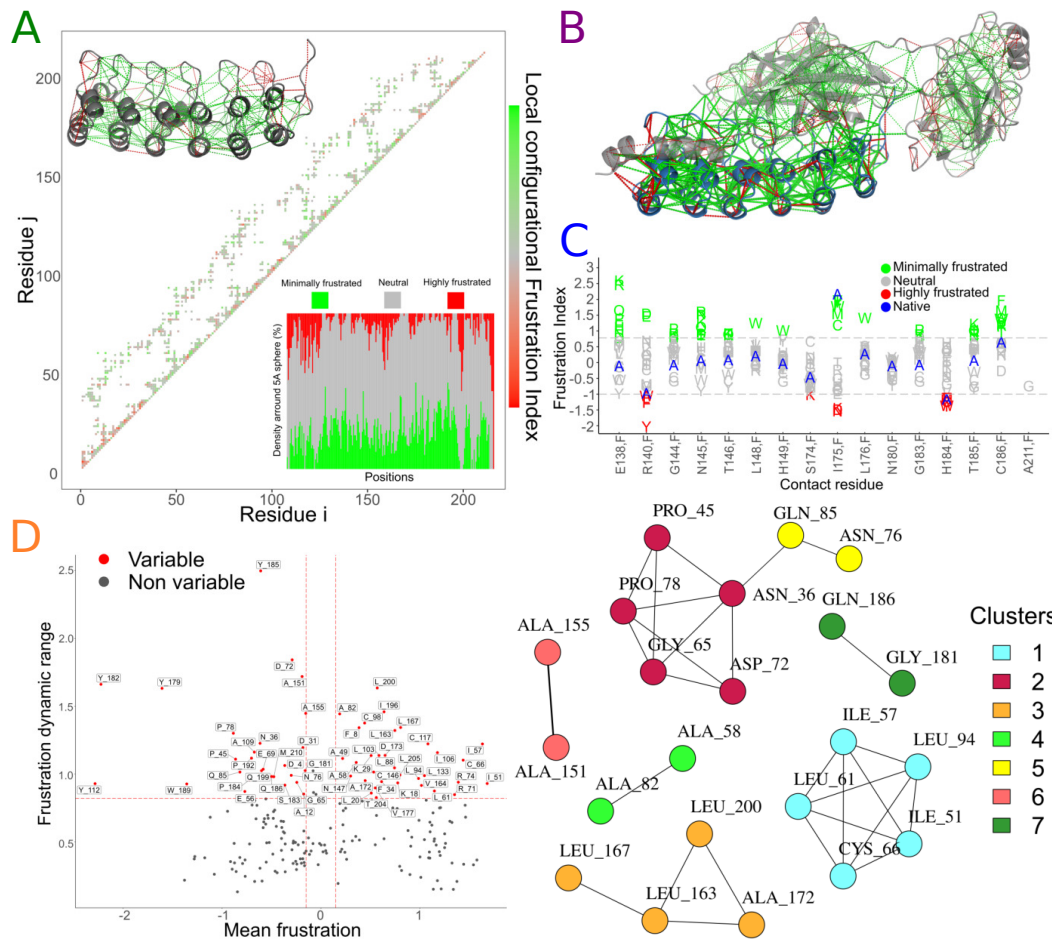
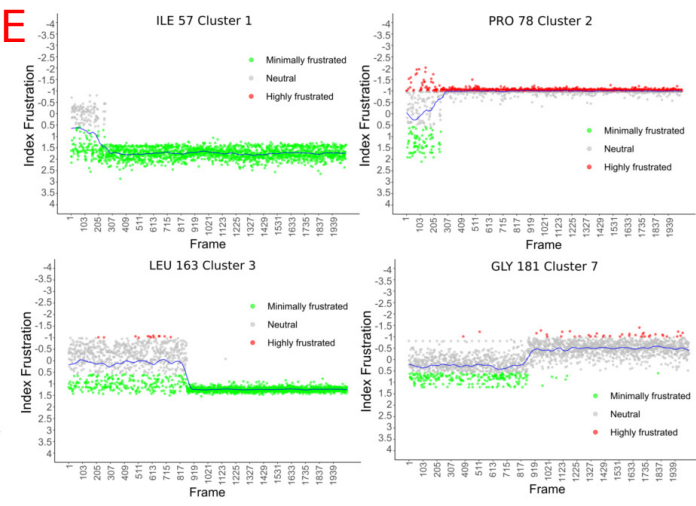

$\mathrm{F}$ library (frustratometeR) plot_contact_map (Pdb_conf) Plot_-5Adens_proportions (Pdb_conf)
view_frustration_pymol (Pdb_conf)

Complex $<-$ calculate_frustration $\left(\right.$ PdbID $=" 1$ nfi", Chain $\left.=c\left(" A^{\prime \prime}, " B^{\prime \prime}, " F^{\prime \prime}\right)\right)$ view_frustration_pymol (Complex) Pdb_conf <- mutate_res $\left(\right.$ Pdb $=$ Pdb_conf, Resno $=178$, Chain $\left.=" \mathrm{~F}^{\prime \prime}\right)$ plot_mutate_res $\left(\right.$ Pdb $\bar{c}=$ Pdb_conf, $\overline{R e s n o}^{-}=178$, Chain $\left.=" F^{\prime \prime}\right)$ DynamicFrust <- dynamic_frustration (PdbsDir $=$ PdbsDir) plot_variable_res_filter (Dynamic $=$ DynamicFrust)

DynamicFrust <- dynamic_res (DynamicFrust, Resno $=57$, Chain $=" \mathrm{~F}^{\prime \prime}$ ) plot_res_dynamics (Dynamic $=$ DynamicFrust, Resno $=57$, Chain $\left.=" F^{\prime \prime}\right)$ Repeat to analyze residues 78,163 and 181.

Fig. 1. Frustration in $I \kappa \mathrm{B} \alpha$ (PDB ID $1 \mathrm{nfi}, \mathrm{F})$. (A) Contact map, $5 \mathrm{Adens}$ plot and pymol representation. (B) Frustration of $I \kappa B \alpha$ in complex with $\mathrm{Nf} \kappa \mathrm{B}$ (chains $\mathrm{A}, \mathrm{B}, \mathrm{F})$. (C) Frustration changes when mutating a specific residue to all canonical amino acids alternatives. $X$ axis: all residues that interact with the residue of interest for all mutants are displayed. Y axis: frustration values are shown and coloured based on their frustration classification. Native variant appears in blue. Each mutant is represented by its 1-letter amino acid code to identify to which variant it corresponds. (D) Dynamic frustration modules. Left: Residues that vary their frustration across frames in molecular dynamic simulations are identified based on their frustration average and dynamic range values. Right: Variable residues are connected to each other in a correlation network that is then clustered to find modules with similar dynamic behaviour (details in supplementary material). (E) Frustration values as a function of time (simulation frames) for representative residues in Clusters 1-4. (F) Minimum code to generate the previous panels

energetic optimization by generating an homology model with Modeller (18)). Subsequently, frustration for each mutant is calculated as with calculate_frustration().

Frustration along MD simulations: The $d y$ namic_frustration() function analyses frustration along a molecular dynamics trajectory (Fig1D). The detect_dynamic_clusters() function can group protein residues based on their temporal "singleresidue" frustration profiles and find dynamic modules constituted by highly correlated residues (Fig1D). Individual residues temporal dynamics can be visualized as well (Fig1E).

\section{Discussion}

As an example, we have applied FrustratometeR to $\mathrm{I} \kappa \mathrm{B} \alpha$, an inhibitor of $\mathrm{Nf} \kappa \mathrm{B}$ (PDBID 1nfi). Fig1A shows a composite figure of typical Frustratometer web-server-like visualisations (17) for $\mathrm{I} \kappa \mathrm{B} \alpha$ (chain $\mathrm{F}$ ). Fig1B shows frustration results for $\mathrm{I} \kappa \mathrm{B} \alpha$ in complex with $\mathrm{Nf} \kappa \mathrm{B}$ (chains $\mathrm{A}, \mathrm{B}, \mathrm{F}$ ). Bear in mind that frustration results differ when chains from quaternary complexes are analysed in isolation or in complex due to the existence of compensating interactions between interacting partners. Far from being a disadvantage, differences between monomers and quaternary complexes frustration results can be (and have been) exploited to analyse interaction mechanisms (7). FrustratometeR introduces a new functionality to evaluate the change in frustration for amino acid variants which can be used as a guide to tune specific residue- residue interactions in the structure. Fig1C shows the change in the configurational frustration index for residue ALA178 when it is mutated to all the other amino acids in threading mode. FrustratometeR also includes a module to analyse molecular dynamics simulations and to identify residues that have similar dynamics. We extracted frames from an $\mathrm{I} \kappa \mathrm{B} \alpha$ coarse-grained folding simulation (see supplementary data for details). First, $m$ variable residues are selected based on their average and the dynamic range of "singleresidue" frustration values across frames. A low dimensional representation of a matrix of $m$ residues and $n$ frames is obtained using Principal Components Analysis (PCA) and residues pairwise correlations are calculated to create a graph that is clustered to define dynamic modules (Fig1D). Here we have analysed the trajectory by detecting clusters with adjusted parameters (CorrType $=$ "spearman", FiltMean $=0.15$, MinFrstRange $=$ 0.7 , MinCorr $=0.95$; see supplementary material for more detailed explanations). Although a detailed analysis of the folding mechanism of the $\mathrm{I} \kappa \mathrm{B} \alpha / \mathrm{Nf} \kappa \mathrm{B}$ is beyond the scope of this article, even this simple analysis can give very useful insights about the system. After filtering residues and building a graph with the dynamic residues, seven clusters are left (Fig1D). I $\kappa \mathrm{B} \alpha$ folding is coupled to $\mathrm{Nf} \kappa \mathrm{B}$ recognition, it involves disorder/order transitions and its energy landscape is quite complex (see "detect dynamic clusters" section in supplemental material for an energy landscape representation). In a very condensed fashion, we can say that there are two major transitions: 1) from the unfolded state to an inter- 
mediate state when $\mathrm{I} \kappa \mathrm{B} \alpha \mathrm{N}$-terminal repetitive region starts to interact with $\mathrm{Nf} \kappa \mathrm{B}$ and 2) from the intermediate state to an expanded/folded state when the C-terminal repeats from $\mathrm{I} \kappa \mathrm{B} \alpha$ get structured by forming further stable interactions with $\mathrm{Nf} \kappa \mathrm{B}$ (19). Clusters 1 and 2 involve residues that have a frustration change during the first folding transition (around frame 200). Cluster 1 residues change their state from being neutral or highly frustrated to become minimally frustrated and are mostly located within helices in repeats 1 and 2. Cluster 2 residues change their state from being minimally frustrated to become highly frustrated. These residues are involved in the interactions with $\mathrm{Nf} \kappa \mathrm{B}$ and become frustrated because the structure is forced to adopt its quaternary structure conformation without $\mathrm{Nf} \kappa \mathrm{B}$ being present to energetically compensate them. Clusters 3 and 7 behave much like clusters 1 and 2 but their frustration changes occur during the second folding transition (around frame 850). A more detailed analysis is made in the detect dynamic clusters section in supplemental material.

\section{Conclusion:}

We present a user-friendly $\mathrm{R}$ package to calculate energetic local frustration in protein structures. The package includes new features to assess the effect of mutations in local frustration as well as to analyse frustration along molecular dynamics trajectories. Its simple interface together with the newly implemented functionalities will facilitate frustration analysis at larger scales and can be used to include FrustratometeR as part of different pipelines for protein structural analysis.

\section{Bibliography}

1. JD Bryngelson and PG Wolynes. Spin glasses and the statistical mechanics of protein folding. Proc. Natl. Acad. Sci. U. S. A., 84(21):7524-7528, November 1987.

2. DU Ferreiro et al. Frustration in biomolecules. Quarterly reviews of biophysics, 47(4):285363, 2014.

3. R Espada et al. The design of repeat proteins: Stability conflicts with functionality, 2017.

4. DU Ferreiro et al. Localizing frustration in native proteins and protein assemblies. Proc. Natl. Acad. Sci. U. S. A., 104(50):19819-19824, December 2007.

5. DU Ferreiro et al. On the role of frustration in the energy landscapes of allosteric proteins. Proc. Natt. Acad. Sci. U. S. A., 108(9):3499-3503, March 2011.

6. MI Freiberger et al. Local frustration around enzyme active sites. Proc. Natl. Acad. Sci. U. S. A., 116(10):4037-4043, March 2019.

7. RG Parra et al. Structural and energetic characterization of the ankyrin repeat protein family. PLoS Comput. Biol., 11(12):e1004659, December 2015.

8. Onur Serçinoğlu and Pemra Ozbek. Sequence-structure-function relationships in class $\mathrm{i}$ mhc: A local frustration perspective. PloS one, 15(5):e0232849, 2020.

9. S Kumar et al. Localized structural frustration for evaluating the impact of sequence variants. Nucleic Acids Res., 44(21):10062-10073, December 2016.

10. Lukas $\mathrm{S}$ Stelzl et al. Local frustration determines loop opening during the catalytic cycle of an oxidoreductase. Elife, 9:e54661, 2020.

11. Ida Lindstrom and Jakob Dogan. Dynamics, conformational entropy, and frustration in protein protein interactions involving an intrinsically disordered protein domain. ACS chemical biology, 13(5):1218-1227, 2018.

12. Jing Li et al. Genetically tunable frustration controls allostery in an intrinsically disordered transcription factor. Elife, 6:e30688, 2017.

13. Maria Ines Freiberger et al. Frustration in protein complexes leads to interaction versatility. BioRxiv, 2020.

14. Sebastian Hiller. Chaperone-bound clients: the importance of being dynamic. Trends in biochemical sciences, 44(6):517-527, 2019.

15. DU Ferreiro et al. Frustration, function and folding. Curr. Opin. Struct. Biol., 48:68-73, February 2018.

16. M Jenik et al. Protein frustratometer: a tool to localize energetic frustration in protein molecules. Nucleic Acids Res., 40(Web Server issue):W348-51, July 2012.

17. RG Parra et al. Protein frustratometer 2: a tool to localize energetic frustration in protein molecules, now with electrostatics. Nucleic Acids Res., 44(W1):W356-60, July 2016.

18. Benjamin Webb and Andrej Sali. Comparative protein structure modeling using modeller. Current protocols in bioinformatics, 54(1):5-6, 2016.

19. Diego U Ferreiro and Elizabeth A Komives. Molecular mechanisms of system control of nf- $\kappa \mathrm{b}$ signaling by $\mathrm{i} \kappa \mathrm{b} \alpha$. Biochemistry, 49(8):1560-1567, 2010. 